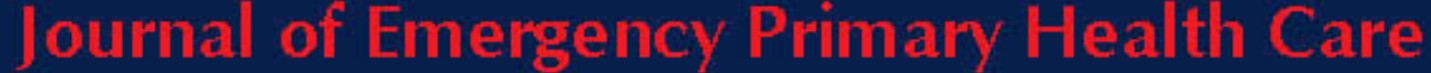

An International elournal of Prehospital Care Research, Education, clinical Practice, Policy and Service Delivery

ISSN 1447-4999

\title{
EDITORIAL
}

\section{Standards and Standardization in Paramedic Protocols}

Article No. 990075

\section{Associate Professor Gaevan Anantharaman}

Patient care protocols have been introduced into emergency pre-hospital care gradually over the last four decades in various ways. While protocols were initially designed to allow a move towards greater indirect medical oversight of paramedic-based emergency ambulance care, the formats, standards and content of such protocols have all developed independently. In the early days, providing avenues for the development of different formats of protocols, and even variations in content, resulted in differences in standards of the care provided by paramedics. This was to be expected. Therefore, the development of different paramedic systems led to varying standards of care in different pre-hospital emergency medical communities and in different countries. Such care has varied from basic first aid to advanced first aid provided by ambulance-based first responders, up to delegated medical acts that some critical care trained paramedics perform.

With the rapid improvement in telecommunications, electronics, computers, the web, faster international travel and greater sharing of ideas, knowledge of the existence of such varying standards became commonplace. The gradual growth of evidential medicine, including in the pre-hospital emergency care environment, is leading to efforts to base more paramedic protocols on existing evidence and towards the conduct of a greater number of randomized controlled trials in this area. It is also leading to the realization that the basis for performing additional advanced paramedic procedures should not be because such a skill may be useful, but more often, that such interventions are proven to demonstrate benefit.

Protocols for pre-hospital emergency care were drawn up for the following reasons: To allow medical assessment and care to begin prior to the arrival of the patient at the hospital facility.

- To define the basis for the various emergency care procedures carried out in the prehospital environment.

- To work towards standards for provision of pre-hospital emergency care by paramedics.

- To inform and familiarize receiving hospital staff with the details of care provided in ambulances and thus reach out into the community.

- To provide a framework for the implementation of medical oversight of care in ambulances - whether provided directly or indirectly.

Sometimes, colleagues in other medical disciplines have presumed that such protocols are a replacement for direct, “on-line” medical oversight. Effort is required to address this misconception. Such misconceptions become greater especially when a great degree of non- 
uniformity is noticed amongst format and content of paramedic protocols amongst various emergency ambulance services.

Our paramedic colleagues have, sometimes, presumed that structured paramedic protocols are an alternative reference or teaching manual for emergency ambulance crew. It is not, then, clearly appreciated that such protocols form the desired standard of care in the ambulance services. Why then, do they ask, does there need to be major differences in format, presentation and content of such protocols amongst the EMSs of different communities?

Protocols, sometimes, run counter to the wishes of the patient or family especially when evacuation is to the nearest hospital, rather than the patient's hospital of choice. Comparisons are occasionally made with those of other services, and a large number of such protocols and formats are available on the web and accessed by members of the public to allow such comparison..

The science that underlies the content of paramedic protocols is now universally known and easily accessed through modern electronic technology. There is increasing evidence that is being unearthed in the medical literature for the use or non-use of various drugs, procedures and old practices in the emergency pre-hospital environment. In such a rapidly evolving environment, there is a need to work towards consensus on formats, organisation and content in the development of these emergency care protocols.

Paramedic protocols, today, come in a variety of forms. Many EMS organisations use three groups of protocols, viz. clinical protocols, patient procedures and drug monographs. An expressed criticism of the tripartite style has been that these tend to perpetuate the perception of these being replacement teaching or reading material for ambulance crew, and an alternative to textbooks on the topics. Some services have kept the number of protocols to a small number that reflects the areas they intend to audit on a regular basis. Others have built up large numbers of such protocols, one for each of the large variety of medical problems that may be seen amongst the patients handled by the particular ambulance service.

Formats and styles adopted have also seen a great degree of variance. Many communities use lists of standing operating procedures for a variety of care protocols. These standing operating procedures are usually bulleted or numbered, the length of each such protocol varying from just about half a page to a few pages. Some are in prose and resemble short monographs on the topic, which, not surprisingly, results in them being used as alternate textbooks for paramedic teaching and training.

Some communities have adopted the algorithmic approach, with the first page of any protocol being presented in the form of an algorithm with each component being numbered, and the decision tree having a combination of rectangles and diamonds to signify treatment approaches and decision points. Such algorithms are often followed by numbered notes that explain the content, reasons for choices made, and precautions to be taken at each step of the decision tree.

Such a structured approach, while seeming prescriptive, has been able to offer the paramedic choices depending on the presentation of the patient, rather than demanding that a definite diagnosis made before care is provided.

Over the last few years there have been some efforts to decrease the variations in format and content. Such standardization is gradually being considered in states with multiple county EMS organisations so that uniformity of state-of-art standards of care are seen to be applied across the larger community. Country-wide standardization and standardization across 
different countries has yet to begin. As communities become more litigious, comparisons become inevitable, and the demands for standardization grow.

The need to standardize would, naturally, lead to moves to establish common, comparable lists of paramedic competencies based on well-documented skill sets. This will be likely to ensure standardization of definitions of such competencies and of the modes of care that should be made available. In turn, this has to result in common approaches to the evaluation and treatment of a host of identified problems seen in everyday paramedic practice. Such a result requires medical directors of EMS programs to get together, and to begin to agree on common approaches, formats and content. It requires a strong degree of bonding amongst such medical directors. Whether such bonding and common approaches should be mandated by federal authorities or come as a result of natural affinity amongst medical directors can be an area of contention.

The advantage of striving towards common standards and common, shared protocols across paramedic services can lead towards integration of pre-hospital services, which while promoting greater communication amongst the various EMS programs within a community, may not be always welcomed by all. Such issues become more difficult to deal with when we begin to address similar issues across national boundaries. In the long run, it may be wiser for the EMS community, and especially the medical directors of such programs to remember that failure to achieve common standards across communities would be more likely to lead to greater public pressures to standardize. The question that would then surface would be whether the public and the state should dictate the integration process.

What is becoming increasingly clear is that the clinical practices of paramedics in the prehospital environment should, more frequently, be evidence-based. Medical Directors across communities within states and also across countries should come together to agree on common standards of care, common formats and common content, before the public applies such pressure. State support for such standardization would lend weight to these efforts. The intention should be to move towards greater integration of pre-hospitals emergency care services across the county and across national boundaries, where these are relevant and appropriate. All these will greatly assist to place the science of pre-hospital emergency care on a level that will enhance acceptance and compliance.

A/Prof Anantharaman is Chairman, Division of Ambulatory \& Clinical Support Services at Singapore General Hospital, and Clinical Associate Professor at the National University of Singapore. 